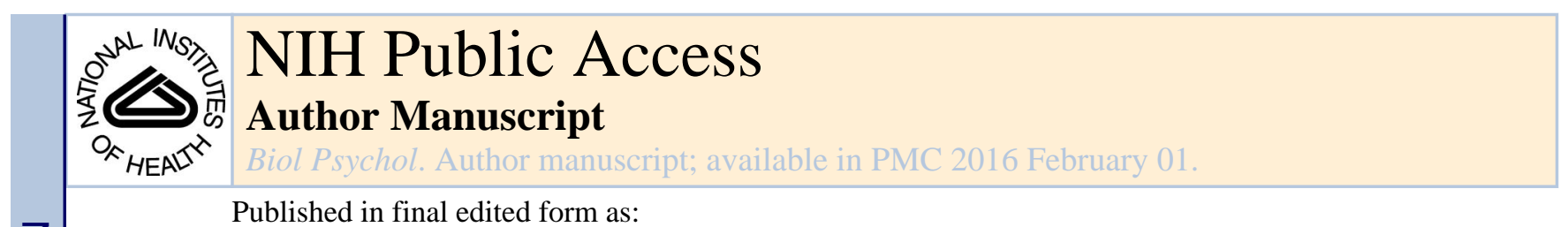

Published in final edited form as:

Biol Psychol. 2015 February ; 0: 124-129. doi:10.1016/j.biopsycho.2015.01.005.

\title{
Allostatic Dysregulation of Natural Reward Processing in Prescription Opioid Misuse: Autonomic and Attentional Evidence
}

\author{
Eric L. Garland, PhD, \\ University of Utah \\ Brett Froeliger, $\mathrm{PhD}$, and \\ Medical University of South Carolina \\ Matthew O. Howard, PhD \\ University of North Carolina at Chapel Hill
}

\begin{abstract}
Chronic pain patients who misuse prescription opioids may suffer from allostatic dysregulation of natural reward processing. Hence, this study examined whether prescription opioid misusers with chronic pain $(n=72)$ evidenced decreased natural reward responsiveness relative to non-misusers with chronic pain $(\mathrm{n}=26)$. Subjects completed a dot probe task containing pain-related, opioidrelated, and natural reward stimuli while attentional bias (AB) scores and heart rate variability (HRV) responses were assessed. Compared to non-misusers, misusers evidenced significantly more attenuated HRV responses to opioid, pain, and natural reward cues presented during the dot probe task. These significant between-groups differences in HRV were largest during attention to natural reward cues, but became non-significant in a sensitivity analysis controlling for opioid dosing. In addition, non-misusers evidenced an $\mathrm{AB}$ toward natural reward cues, whereas misusers did not. Findings suggest that opioid misusers exhibit attentional and autonomic deficits during reward processing.
\end{abstract}

\section{Keywords}

opioid misuse; heart-rate variability; attentional bias; reward processing; chronic pain

Chronic exposure to prescription opioid analgesics may result in opioid misuse and addiction due to the pharmacologic actions of opioids on the mesocorticolimbic dopamine system - the common neural circuitry underlying a broad range of addictive behaviors (Koob \& Volkow, 2009). Chronic pain patients prescribed long-term opioid analgesic pharmacotherapy are at risk for developing prescription opioid use disorder; approximately

C 2015 Published by Elsevier B.V.

Corresponding author: Eric L. Garland, 395 South, 1500 East, University of Utah, Salt Lake City, UT 84112, elgarlan@gmail.com.

Publisher's Disclaimer: This is a PDF file of an unedited manuscript that has been accepted for publication. As a service to our customers we are providing this early version of the manuscript. The manuscript will undergo copyediting, typesetting, and review of the resulting proof before it is published in its final citable form. Please note that during the production process errors may be discovered which could affect the content, and all legal disclaimers that apply to the journal pertain. 
$10 \%$ of such individuals exhibit opioid misuse behaviors such as unauthorized dose escalation or self-medication of negative affect with opioids (Fishbain, Cole, Lewis, Rosomoff, \& Rosomoff, 2007). A recent survey of treatment-seeking opioid dependent persons found rates of opioid self-medication of negative affective states as high as $90 \%$ (Garland, Hanley, Thomas, Knoll, \& Ferraro, in press). Thus, prescription opioid misusers may seek and consume opioids as a means of regulating dysphoria and maintaining a positive hedonic tone.

Maintenance of hedonic homeostasis may be undermined by increasing tolerance to the effects of opioids coupled with recurrent episodes of chronic pain and coincident negative emotions. The individual may thereby be impelled to use increasingly higher doses of opioids to allay emotional and somatic distress (Shurman, Koob, \& Gutstein, 2010). However, this attempt to achieve an equilibrium is costly: chronic opioid use may shift the hedonic set point, rendering the individual increasingly insensitive to rewards in the natural environment and tipping the hedonic balance further toward negative affectivity (Koob \& Le Moal, 2008). In turn, the effort to preserve dwindling hedonic tone may fuel a cycle of escalating dependence on opioids (Garland, Froeliger, Zeidan, Partin, \& Howard, 2013a).

Pre-clinical and clinical studies suggest that addiction to a wide range of substances is undergirded by changes in dopaminergically-mediated reward function (Augustus Diggs, Froeliger, Carlson, \& Gilbert, 2013; Gipson et al., 2013; Heinz et al., 2004; Kalivas \& Volkow, 2005; Lintas et al., 2012) including attenuated neurobehavioral reactivity to natural rewards and heightened reactivity to drug-related cues (Koob \& Volkow, 2009; Volkow, Wang, Fowler, Tomasi, \& Telang, 2011). In contradistinction to healthy, non-addicted individuals who exhibit an attentional bias $(\mathrm{AB})$ toward images representing positive, naturally rewarding stimuli (Wadlinger \& Isaacowitz, 2010), opiate dependent individuals evidence an $\mathrm{AB}$ to opiate-related visual cues presented for $500 \mathrm{~ms}$ on dot probe tasks, as evidenced by faster responses to probes replacing opiate photographs than to probes replacing neutral probes (Lubman, Peters, Mogg, Bradley, \& Deakin, 2000). Similarly, prescription opioid dependent chronic pain patients exhibit an $\mathrm{AB}$ to prescription opioid cues presented for $200 \mathrm{~ms}$ (Garland, Froeliger, Passik, \& Howard, 2013b). AB for a cue presented for $\leq 200 \mathrm{~ms}$ is believed to index biases in initial attentional orienting, whereas $\mathrm{AB}$ for longer duration stimuli (> $500 \mathrm{~ms}$ ) is believed to index delayed disengagement of attention from an emotionally salient cue (Field \& Cox, 2008). Moreover, opiate dependent individuals exhibit enhanced event-related brain potentials (e.g., P300) to opiate cues coupled with attenuated electrophysiological brain responses to images depicting natural rewards (Lubman, Allen, Peters, \& Deakin, 2007, 2008). Such decreased responsiveness to natural rewards is a robust predictor of future opiate use (Lubman et al., 2009). Although the studies by Lubman and colleagues support the presence of decreased responsiveness to natural rewards among individuals addicted to illicit opiates (e.g., heroin), to our knowledge no study has identified reward dysfunction among chronic pain patients who misuse prescription opioids. If research demonstrates the presence of deficits in natural reward processing among such patients, such a finding would provide a potentially crucial treatment target and help to further elucidate the risk chain leading from chronic pain to opioid misuse and addiction (for a review, see Garland et al., 2013a). 
Attentional and emotional processing of rewarding or emotionally-salient cues is thought to elicit reactivity in a network of central (e.g., prefrontal cortex [PFC], anterior cingulate cortex $[\mathrm{ACC}]$ ) and autonomic nervous system structures with downstream effects on visceral and peripheral parameters, including the beat-to-beat modulation of heart rate by the vagus nerve, known as high-frequency heart rate variability (HRV) (Thayer \& Lane, 2000, 2009). HRV is mediated by parasympathetic influences on the sinoatrial node of the heart (Berntson et al., 1997). Elevated HRV may reflect self-regulatory effort or efficacy (Segerstrom \& Nes, 2007), and individuals with impairments in regulation of attention, emotion, and appetitive urges exhibit attenuated HRV at rest (Ingjaldsson, Laberg, \& Thayer, 2003a; Thayer \& Lane, 2009) and when attempting to suppress craving in response to addiction-related cues (Garland, Carter, Ropes, \& Howard, 2012). Yet, increased HRV can also be elicited as a classically conditioned response to conditioned appetitive stimuli (Inagaki, Kuwahara, \& Tsubone, 2005; Stockhorst, Huenig, Ziegler, \& Scherbaum, 2011). Studies have identified cue-elicited increases in HRV associated with craving for addictive substances such as methamphetamines, nicotine, and alcohol (Culbertson et al., 2010; Erblich, Bovbjerg, \& Sloan, 2011; Garland, Franken, Sheetz, \& Howard, 2012; Garland, Franken, \& Howard, 2012; Ingjaldsson, Laberg, \& Thayer, 2003; Rajan, Murthy, Ramakrishnan, Gangadhar, \& Janakiramaiah, 1998), and increased HRV during exposure to food cues (Udo et al., 2013) which abates upon consumption of a meal (Nederkoorn, Smulders, \& Jansen, 2000). Thus, HRV may be a useful index of self-regulation and reward responsiveness among prescription opioid misusing chronic pain patients, though no study has examined this measure in this clinical population to date.

To address the dearth of findings in this potentially important research area, the present study aimed a) to establish whether prescription opioid misusers with chronic pain evidence decreased natural reward responsiveness (as indicated by HRV responses) relative to chronic pain patients who take opioids as medically prescribed and $b$ ) to determine whether prescription opioid misusers with chronic pain exhibit comparatively attenuated cardiacautonomic control during attention to a range of emotionally-salient cues. As converging evidence of reward dysregulation, we sought to determine if opioid misuse was associated with reduced $\mathrm{AB}$ to natural reward cues.

To examine these questions in the present study, a sample of opioid-misusing chronic pain patients (opioid misusers) and chronic pain patients who did not misuse opioids (nonmisusers) completed a dot probe task in which opioid-related, pain-related, and naturalreward images were presented while HRV was measured concurrently. We had three hypotheses: 1) as an index of impaired cardiac-autonomic control during regulation of attention to emotional information, opioid misusers would exhibit significantly less phasic cue-elicited HRV during the dot probe task than non-misusers, but would not show differences in resting state HRV at baseline; 2) given that deficits in natural reward processing are a hallmark of addiction, these phasic HRV differences would be most pronounced for natural reward-related cues; and 3) as further evidence of deficient reward processing, we also hypothesized that the non-misuser group would exhibit a significant positive $A B$ towards natural reward cues, whereas the opioid misuser group would not exhibit this normative positive $\mathrm{AB}$ - indicative of their underlying reward deficit. 


\section{Methods}

\section{Participants}

Participants met eligibility criteria if they had a diagnosable chronic pain condition, had been prescribed long-term analgesic pharmacotherapy (for at least 90 days, see clinical guidelines presented in Chou et al., 2009), and had taken opioids daily or nearly every day ( $\geq 5$ days/week) for $>3$ months. Participants were recruited from primary care clinics, pain clinics, and neurology clinics in Tallahassee, FL via flyers and online classified ads. Advertisements sought to recruit participants who "suffer from and are prescribed medicine for chronic pain" for a study focused on improving ways to address problems with chronic pain and prescription pain medication. Prescription opioid misuse was determined by scores on the self-reported Current Opioid Misuse Measure (COMM; $\alpha=.83$ ) (Butler et al., 2007). The original COMM validation study conducted with patients treated in specialty pain management clinics found that a score of $\geq 9$ was suggestive of prescription opioid misuse. However, according to a study of a broad sample of chronic pain patients from a variety of primary care settings who took prescription opioids but not necessarily on a daily basis, receiver-operator characteristic curve analyses revealed that a score of 13 or higher on the COMM had maximum sensitivity and specificity to identify prescription opioid misuse among chronic pain patients in primary care settings (Meltzer et al., 2011). We chose this more conservative COMM threshold value to minimize false positives and because, similar to Meltzer et al. (2011), our sample was broad and not confined to patients from specialty pain clinics.

Based on this cutoff score, participants were grouped into one of two groups: a group of chronic pain patients who took prescription opioids daily/nearly every day and reportedly engaged in opioid misuse behaviors (misusers, $\mathrm{n}=72$ ), and a group of chronic pain patients who took prescription opioids daily/nearly every day without engaging in opioid misuse (non-misusers, $\mathrm{n}=26$ ). Table 1 describes participant demographics and prevalence of various chronic pain conditions in the sample.

\section{Data Collection}

Current opioid misuse measure-The Current Opioid Misuse Measure (COMM; $a=$. 83) (Butler et al., 2007) assessed self-reported aberrant drug-related behavior. Participants responded to 17 items rated on a Likert scale $(0=$ never, $4=$ very often $)$ regarding how often in the past 30 days they had engaged in behaviors potentially reflective of opioid misuse or took opioid medication in excessive doses or in nonprescribed ways, tapped by items such as "In the past 30 days, how often have you taken your medications differently from how they are prescribed?", "In the past 30 days, how often have you used your pain medicine for symptoms other than for pain (e.g., to help you sleep, improve your mood, or relieve stress)?"

Dot probe task-A dot probe task was used to measure $\mathrm{AB}$ to opioid-related, pain-related, and natural reward cues. Each trial began with a fixation cross (i.e., crosshair) presented for $500 \mathrm{~ms}$. Next, two images matched for visual complexity, composition, and figure-ground relationships appeared side by side on the computer screen for either 200 or $2000 \mathrm{~ms}$. Pairs 
of photos containing one emotionally-salient image and one neutral image were presented. Three blocks of cues (opioid-related, pain-related, and pleasure-related) were presented in randomized, counterbalanced order across participants. Specific picture cues were presented in a randomized order within each block and blocks were counterbalanced across participants.

Three sets of 12 photographs, each set representing one type of cue, were selected from the International Affective Picture System (IAPS) (Lang, Bradley, \& Cuthbert, 1997) and media libraries on the Internet. Opioid-related cues included images of pills and pill bottles. Painrelated cues included images of severe injuries, painful medical procedures, and human faces grimacing in pain. Natural reward cues included images of romantic couples, athletic victories, and food. A set of 36 neutral images was selected from the IAPS and each neutral image was paired with an emotionally-salient image matched for visual features such as color, figure-ground relationships, and presence of human faces.

Presentation duration and left/right position of the images were randomized and counterbalanced within each block of 64 trials. Half $(n=32)$ of the trials for each cue type were presented in each visual field (VF: left VF, right VF), and within each VF, half of the trials were presented at each presentation duration $(200 \mathrm{~ms}, \mathrm{n}=16 ; 2000 \mathrm{~ms}, \mathrm{n}=16)$. The image pairs disappeared, and a target probe replaced one of the images after a $50 \mathrm{~ms}$ interstimulus interval (ISI). Probes appeared for $100 \mathrm{~ms}$, and probe location (left VF, right VF) was counterbalanced. Each block was presented 1 time, and the order of blocks over the timecourse of the task was counterbalanced across participants. Participants indicated the location of the target by responding with a left/right button press, and the reaction time (RT) was recorded.

HRV Measurement-Disposable $\mathrm{Ag}-\mathrm{AgC} 1$ electrodes were attached to participants' right and left pectoral muscles. Electrocardiogram (ECG) data were sampled at $1000 \mathrm{~Hz}$ and recorded continuously throughout the protocol on a Biopac MP150 (Biopac Systems, Goleta, CA). Respiration rate was concomitantly assessed with a breathing belt and also recorded on the Biopac MP 150 system.

\section{Procedures}

Participants were instructed to take their prescribed opioid medication as usual on the day they completed study measures. In a single session, participants first completed the Current Opioid Misuse Measure, and next were instructed to remain motionless and silent for a 5minute baseline, after which they participated in the dot probe task (which took approximately 15 minutes to complete). All participants provided written, informed consent and were compensated $\$ 25$ for their participation in the study. The study was approved by the Human Subjects Committee of Florida State University and comports with ethical provisions set in the Declaration of Helsinki.

\section{Data Reduction}

Regarding the analysis of $\mathrm{AB}$ data for each participant, trials with extreme RTs, defined as those with RTs 3 SD above or below the individual mean RT (Field, Mogg, Zetteler, \& 
Bradley, 2004; Ratcliffe, 1993), were eliminated as outliers ( $<2 \%$ of trials were discarded). Trials on which the probe location was incorrectly identified were also omitted in $\mathrm{AB}$ analyses (misusers $15.3 \% \pm 1.7 \%$; non-misusers $9.9 \% \pm 2.9 \%$ ) - there was no significant between-groups difference in accuracy on the task. Opioid, pain, and natural reward AB scores were calculated by subtracting participants' mean RT to target probes replacing emotionally salient photos (opioid, pain, or natural reward cues) from their mean RT to target probes replacing neutral photos, such that positive bias scores indicate an AB toward emotionally salient cues. One-sample Kolmogorov-Smirnov tests revealed that the distributions of the $200 \mathrm{~ms}$ AB scores and $2000 \mathrm{~ms}$ AB scores did not significantly differ from a normal distribution.

With respect to the HRV analyses, R-R intervals were detected in the ECG data using automated routines in Acqknowledge 4.1 software (BIOPAC, Inc.). The R-wave file was then visually inspected to correct misidentified or omitted R-waves. Kubios 2.0 (Biosignal Analysis and Medical Imaging Group, University of Finland) was used to calculate beatsper-minute (BPM) and for spectral analysis of R-waves. R-R interval data was segmented into the following windows: the 5-minute baseline, the opioid cue block, the pain cue block, and the natural reward cue block. A fast Fourier transform was applied separately to R-R interval data in each time window to extract normalized high-frequency HRV from a detrended, end-tapered interbeat interval time series. We used standard default spectrum estimation settings in Kubios prior to applying the Fourier transform (window width: 256s, window overlap: 50\%). High-frequency HRV in the respiratory frequency band $(0.15-0.40$ $\mathrm{Hz}$ ) was selected as our estimate of vagally-mediated HRV. Heart rate variability frequency measures can allow for disentanglement of predominately parasympathetically-mediated HRV (i.e., high-frequency HRV) from HRV driven by parasympathetic and sympathetic influences (i.e., low-frequency HRV) (Berntson, 1997; Malliani, Lombardi, Pagani, 1994). Following Berntson (1997) and Malliani et al. (1994), we calculated high-frequency in normalized units to elucidate shifts in this frequency component that might otherwise be obscured by use of absolute units which are dependent on total HRV power (including very low frequency HRV). HRV was averaged for each of the dot probe cue blocks separately, and was also averaged across the 5-minute baseline. Because there was no significant Group $(F(1,84)=1.14, p=.29)$ or Group $\times$ Condition difference $(F(1,84)=.19, p=.95)$ in respiration rate between misusers and non-misusers, we did not control for respiration rate in our HRV analyses. HRV data were skewed and log-transformed for subsequent analyses.

\section{Statistical Analysis}

For hypothesis testing, we used the following multistage analytic approach. To test hypothesis 1, we first conducted a One-Way ANOVA to examine differences in resting state HRV between misusers and non-misusers. Next, we conducted a two-way Repeated Measures-ANOVA (RM-ANOVA) on the phasic HRV data with Group (misusers, nonmisusers) and Condition (baseline, opioid cue block, pain cue block, pleasure cue block) as factors. In this ANOVA, the main effect of Condition tested to what extent HRV differed between each of the dot probe cue blocks and from resting baseline levels (this was followed by planned contrasts to further specify this effect), whereas the Group $\times$ Condition 
interaction term tested whether misusers and non-misusers significantly differed on HRV values at rest and during each of the dot probe cue conditions.

To test hypothesis 2 and assess between-groups differences in phasic, cue-elicited HRV responses, planned contrasts (contrasting HRV during each of the cue conditions with baseline HRV as the reference category) were used to determine whether opioid misusers and nonmisusers significantly differed with regard to HRV increases from resting baseline levels to each of the dot probe cue conditions (with a specific focus on HRV responsivity to natural reward cues). This computation mathematically produces the same results as using a difference score. In sensitivity analyses, we conducted a RM-ANOVA on phasic HRV scores, controlling for potentially confounding variables with known or theoretical effects on HRV, including age, gender, number of years experiencing chronic pain, and morphine equivalent daily opioid dose.

To test hypothesis 3 , we conducted one sample t-tests to identify the presence of significantly nonzero $\mathrm{AB}$ scores for natural reward cues among misusers and non-misusers. Supplementary t-tests tested for the presence of significantly nonzero AB scores for opioid and pain-related cues among misusers and non-misusers. A follow-up ANCOVA using the covariates listed above was used to test between-groups differences in $\mathrm{AB}$.

\section{Results}

Resting-State HRV and Phasic HRV Cue Responses-With regard to hypothesis 1, there was no significant difference in basal, resting-state HRV between the misuser and nonmisuser groups, $F(1,97)=.004, p=.95$. With regard to phasic HRV cue responses, the main effect of Condition was significant, $F(3,96)=4.74, p=.003, \eta^{2}$ partial $=.05$, indicating that HRV differed from baseline to the various cue blocks on the dot probe task. Planned contrasts indicated that this effect was largely driven by significantly increased phasic HRV from baseline to opioid cue exposure across both groups, $F(1,96)=11.62, p=.001, \eta^{2}$ partial $=.11$, though HRV also increased significantly to pain $\left(F(1,96)=4.46, p=.037, \eta^{2}\right.$ partial $=$. $04)$ and pleasure cues $\left(F(1,96)=4.64, p=.034, \eta^{2}\right.$ partial $\left.=.05\right)$. The main effect of Group was marginally significant, $\left(F(1,96)=3.84, \mathrm{p}=.05, \eta^{2}\right.$ partial $\left.=.04\right)$, indicating that opioid misusers evidenced less HRV averaged across all conditions of the protocol than nonmisusers. Crucially, the omnibus Group $\times$ Condition interaction was significant, $F(3,96)=$ $3.51, \mathrm{p}=.02, \eta^{2}$ partial $=.04$. With regard to hypothesis 2 , to clarify this significant interaction, planned contrasts revealed that HRV increased to a significantly greater extent for non-misusers than for misusers from resting baseline to the opioid cue block, $F(1,96)=$ $5.19, p=.03, \eta_{\text {partial }}^{2}=.05$, the pain cue block, $F(1,96)=5.23, p=.03, \eta_{\text {partial }}^{2}=.05$, and the pleasure cue block, $F(1,96)=6.33, p=.01, \eta^{2}$ partial $=06$. These between-groups differences were most pronounced for pleasure cues (see Figure 1).

Sensitivity analysis-In a sensitivity analysis, a RM-ANOVA controlling for age, gender, duration of chronic pain, and morphine equivalent daily opioid dosage indicated that the main effect of Condition was non-significant, $F(3,31)=1.88, p=.14, \eta^{2}$ partial $=.06$, as was the main effect of Group, $F(3,31)=1.68, p=.20, \eta_{\text {partial }}^{2}=.05$. However, the omnibus Group $\times$ Condition interaction was significant, $F(3,31)=3.09, p=.03, \eta^{2}$ partial $=.09$. To 
clarify this significant interaction, planned contrasts indicated that the non-misuser group experienced significantly greater increases in HRV to pain cues compared to the misuser group $\left(F(1,31)=7.06, p=.01, \eta^{2}\right.$ partial $\left.=.19\right)$, but did not differ significantly with regard to HRV response to opioid $\left(F(1,31)=3.52, p=.07, \eta_{\text {partial }}^{2}=.10\right)$ or natural reward cues $\left(F(1,31)=2.38, p=.13, \eta_{\text {partial }}^{2}=.07\right)$.

AB Scores-With regard to hypothesis 3, among the non-misuser group, we observed a significantly nonzero $\mathrm{AB}$ toward natural reward cues presented for $200 \mathrm{~ms}(\mathrm{M}=11.02 \pm$ $4.52 \mathrm{~ms}$; one-sample t-test, $t=2.43, p=.02$ ). Among the misuser group, we observed a nonsignificant $\mathrm{AB}$ away from natural reward cues presented for $200 \mathrm{~ms}(\mathrm{M}=-6.46 \pm 6.83$ $\mathrm{ms}$; one-sample t-test, $t=.35, p>.10$ ). Supplementary analyses revealed that both nonmisusers $(\mathrm{M}=15.39 \pm 6.74 \mathrm{~ms}$; one-sample t-test, $t=2.28, p=.03)$ and misusers had a significant $\mathrm{AB}$ toward prescription opioid cues presented for $200 \mathrm{~ms}(\mathrm{M}=18.18 \pm 4.42 \mathrm{~ms}$; one-sample t-test, $t=4.11, p<.001$ ). In contrast, $\mathrm{AB}$ scores for reward and opioid cues presented for $2000 \mathrm{~ms}$ did not significantly differ from zero, suggesting that $\mathrm{AB}$ was only present during initial attentional orienting and not during sustained attention (Field \& Cox, 2008). In addition, neither non-misusers nor misusers evidenced a significant $A B$ toward pain-related cues at either stimulus duration. A sensitivity analysis controlling for gender, age, duration of pain, and opioid dose revealed no significant between-groups effects on $A B$ scores for any cue type or stimulus duration. AB scores were not significantly associated with resting state HRV or phasic cue-elicited HRV responses.

\section{Discussion}

In this study of chronic pain patients taking prescription opioid analgesics for at least 90days, opioid misusers had significantly more attenuated HRV responses during attention to emotional information than individuals with at low risk for opioid misuse. Indeed, while non-misusers exhibited an increase in HRV from baseline across all three emotional cue types, opioid misusers evidenced comparatively blunted phasic HRV cue responses. This differential autonomic response was most pronounced for natural reward cues. Congruent with this finding, the non-misuser group exhibited a $200 \mathrm{~ms} A B$ to natural reward cues, whereas the misuser group had no such AB. Importantly, there were no significant betweengroups differences in tonic, resting-state HRV. This finding suggests that the neuropharmacologic effects of opioids may not impact tonic, resting-state HRV but may instead inhibit HRV cue responsiveness. Alternatively, this finding may suggest that observed differences in HRV cue responsiveness may have stemmed from the effects of addictive processes on dysregulation of central-autonomic responses.

Across both groups of opioid-using patients, HRV increased significantly from resting-state levels during opioid cue trials. Such increases in HRV cue responses have been observed in preclinical models of appetitive conditioning which indicate that heightened HRV elicited by conditioned stimuli is associated with a reward expecting state (Inagaki et al., 2005), and clinical studies of persons experiencing appetitive responses to addictive drugs (Culbertson et al., 2010; Erblich et al., 2011; Rajan et al., 1998) as well as food cues (Udo et al., 2013). Moreover, increases in alcohol cue-elicited HRV predicted the time-course of relapse among alcohol dependent individuals up to six months following treatment (Garland et al., 2012). 
Thus, phasic HRV elicited by opioid cues may reflect appetitive responding and/or conditioned reward. Supporting this interpretation, both groups of patients exhibited an AB towards opioid cues presented for $200 \mathrm{~ms}$. The $200 \mathrm{~ms} \mathrm{AB}$ is commonly held to index bias in initial attentional orienting (Field \& Cox, 2008). As in the HRV responses to opioid cues, both groups of patients exhibited heightened HRV responses to pain cues, possibly indicating attentional processing of emotionally salient stimuli.

Importantly, non-misusers experienced significantly greater autonomic (i.e., HRV) and attentional (i.e, $\mathrm{AB}$ ) responses to natural reward cues relative to opioid misusers. To our knowledge, this is the first finding in the literature that chronic pain patients who misuse prescription opioids exhibit pronounced reward processing deficits relative to chronic pain patients who take opioids as prescribed. Prior preclinical studies have demonstrated effects of chronic pain on brain reward systems which parallel the clinical observation that chronic pain is often accompanied by significant anhedonia and loss of motivation to obtain reward (Becker, Gandhi, \& Schweinhardt, 2012; Schwartz et al., 2014). Yet, the present study suggests that prescription opioid misuse is associated with additional reward deficits above and beyond the presence of chronic pain. Putatively, such reward deficits drive escalation of opioid misuse as a means of coping with dysphoria stemming from chronic pain and mesocorticolimbic dopamine system dysregulation (Garland et al., 2013a). That is, study findings provide support for the allostatic model of addiction (Koob \& Le Moal, 2001, 2008). The allostatic model posits that prolonged exposure to drug experiences results in an upward shift in basal reward threshold, leading to a reward deficit and dysphoric mood that encourages increased consumption of drugs as a means of achieving an overall positive affective balance. Ironically, this attempt to reach a positive affective state comes with a cost: the continued use of drugs further increases brain reward thresholds, making the individual increasingly insensitive to naturally-rewarding experiences while becoming increasingly sensitive to stress and pain (Koob \& Le Moal, 2001). Although prior studies have identified allostatic dysregulation of reward processing among opiate addicts, the present study provides novel evidence that such allostatic processes may also undergird prescription opioid misuse among chronic pain patients.

It is possible that the reduced reward responsiveness observed among the opioid-misusing patients in this study was a product of the allostatic state induced by chronic high dose opioid exposure; this interpretation is bolstered by the finding that after controlling for differences in opioid dosing, there were no significant between-groups differences in HRV or $\mathrm{AB}$ responses to natural reward and opioid cues. However, we cannot rule out that age, gender, or chronic pain duration might also be responsible for these effects, given that these variables were also controlled in our sensitivity analyses. Indeed, age and gender are known to influence HRV (Antelmi, De Paula, Shinzato, Peres, Mansur, \& Grupi, 2004) and AB towards positive affective stimuli (Wadlinger \& Isaacowitz, 2010). Moreover, AB and HRV have been linked with chronicity of pain (Schoth, Nunes, \& Liossi, 2012; Nes, Roach, \& Segerstrom, 2009) and severity of drug use and/or addiction severity (Field \& Cox, 2008; Garland, Franken, \& Howard, 2012). Given that these factors are known to be associated with attentional and autonomic regulation, it is perhaps unsurprising that the observed between-groups effects on cue-elicited HRV and AB responses did not survive statistical 
correction for duration of chronic pain, drug use severity, gender, and age - by using this set of covariates, we were in effect controlling for factors linked with the outcome of interest.

Importantly, the observed between-groups difference in phasic HRV response to painrelated cues remained significant even after controlling for these factors, suggesting that regardless of chronicity of pain or opioid dosage (as well as age and gender), opioid misusing chronic pain patients exhibit comparatively attenuated parasympathetic response to emotionally threatening stimuli. While cue-elicited increases in HRV to appetitive stimuli are thought to index reward responsiveness (Inagaki et al., 2005), cue-elicited increases in HRV to emotionally aversive stimuli are thought to index self-regulation (Segerstrom \& Nes, 2006) or emotion regulation (Thayer \& Lane, 2000; 2009). Indeed, reduced cue-elicited HRV during emotion regulation has been observed among persons with high neuroticism relative to those with low neuroticism (Di Simplicio, Costoloni, Western, Hanson, Taggart, \& Harmer, 2012). In this light, our findings may imply the presence of self-regulatory deficits among opioid misusing chronic pain patients (Nes et al., 2009), at least with respect to autonomic regulation of homeostatic perturbations elicited by pain-related stimuli. Hypothetically, deficient regulation of negative emotional responses might promote opioid misuse among chronic pain patients by exacerbating craving and maladaptive cognitive processes (Garland et al., 2013a; Martel, Dolman, Edwards, Jamison, \& Wasan, 2014).

Irrespective of the etiology of the observed neurocognitive deficits among opioid misusers in this sample, restructuring reward responsiveness may be a fruitful target for opioid misuse interventions, as evidenced by a recent study which found that the therapeutic effects of a Mindfulness-Oriented Recovery Enhancement intervention on reducing prescription opioid craving were statistically associated with increases in natural reward processing as indicated by cardiac-autonomic responses (Garland, Froeliger, \& Howard, 2014a) and event-related potentials (Garland, Froeliger, \& Howard, 2014b).

The primary limitation of this study was the fact that we assessed a sample of volunteers and that we confined our primary analyses to those subjects who had no missing data. These procedures might have implications for the generalizability of our findings, as patient samples who volunteer for research may significantly differ from those who do not. Also, we characterized patients as opioid misusers based on their score on a self-report instrument (the COMM). Because some patients may be reluctant to admit to opioid misuse on a selfreport measure, reporting bias is possible. Moreover, following Meltzer et al. (2011), we selected a more conservative COMM threshold value than the original COMM validation study (Butler et al., 2007) to minimize false positives. For these reasons, we may not have been able to correctly classify all the misusers in the sample. Nonetheless, participants were assured of confidentiality, and the majority endorsed opioid misuse on this measure even with the more restrictive threshold. Lastly, we instructed participants to take their opioids as prescribed on the day of the dot probe testing session to prevent opioid withdrawal which might have had deleterious effects on participants' health and cognitive performance. Yet, medication use might have influenced performance on the dot probe task. As such, we included morphine equivalent daily dose as a covariate in sensitivity analyses. Although the findings should be considered preliminary and heuristically informative, this exploratory study helps to raise a number of key issues that should be explored in larger and more well- 
controlled studies which couple neuroimaging with autonomic and pharmacologic probes to disentangle the differential contribution to deficient HRV cue responses made by the purely pharmacologic effects of acute opioid exposure from the effects of neural dysfunction associated with addictive processes.

In conclusion, results from this study suggest that opioid-misusing chronic pain patients exhibit attenuated natural reward processing relative to pain patients who take opioids as prescribed. This reward processing deficit may stem from the allostatic state induced by chronic high dose opioid exposure or from the effects of addiction on dysregulation of central-autonomic cue-responses. Regardless of its source, deficient reward processing may be an important risk factor for the development and maintenance of prescription opioid misuse and addiction.

\section{Acknowledgments}

This work was supported by grant numbers DA032517 and DA037005 from the National Institutes of Health awarded to E.L.G.; and a grant from the Fahs Beck Fund for Research and Experimentation, also awarded to E.L.G.

\section{References}

Antelmi I, De Paula RS, Shinzato AR, Peres CA, Mansur AJ, Grupi CJ. Influence of age, gender, body mass index, and functional capacity on heart rate variability in a cohort of subjects without heart disease. The American Journal of Cardiology. 2004; 93(3):381-385. [PubMed: 14759400]

Augustus Diggs H, Froeliger B, Michael Carlson J, George Gilbert D. Smoker-nonsmoker differences in neural response to smoking-related and affective cues: An fMRI investigation. Psychiatry Research: Neuroimaging. 2013; 211(1):85-87.

Becker, S.; Gandhi, W.; Schweinhardt, P. Cerebral interactions of pain and reward and their relevance for chronic pain. Neuroscience Letters. 2012. Retrieved from http://www.sciencedirect.com/science/ article/pii/S030439401200362X

Berntson GG, Bigger JT Jr, Eckberg DL, Grossman P, Kaufman PG, Malik M, van der Molen MW. Heart rate variability: Origins, methods, and interpretive caveats. Psychophysiology. 1997; 34:623648. [PubMed: 9401419]

Butler SF, Budman SH, Fernandez KC, Houle B, Benoit C, Katz N, Jamison RN. Development and validation of the Current Opioid Misuse Measure. Pain. 2007; 130:144-56. [PubMed: 17493754]

Chou R, Fanciullo GJ, Fine PG, Adler JA, Ballantyne JC, Davies P, et al. Clinical guidelines for the use of chronic opioid therapy in chronic noncancer pain. The Journal of Pain. 2009; 10(2):113-130. [PubMed: 19187889]

Culbertson C, Nicolas S, Zaharovits I, London ED, La Garza I, Brody AL, Newton TF. Methamphetamine craving induced in an online virtual reality environment. Pharmacology Biochemistry and Behavior. 2010; 96(4):454-460.

Di Simplicio M, Costoloni G, Western D, Hanson B, Taggart P, Harmer CJ. Decreased heart rate variability during emotion regulation in subjects at risk for psychopathology. Psychological Medicine. 2012; 42(8):1775-1783. [PubMed: 22067596]

Erblich J, Bovbjerg DH, Sloan RP. Exposure to smoking cues: Cardiovascular and autonomic effects. Addictive Behaviors. 2011; 36(7):737-742.10.1016/j.addbeh.2011.02.011 [PubMed: 21419576]

Field M, Cox WM. Attentional bias in addictive behaviors: a review of its development, causes, and consequences. Drug and Alcohol Dependence. 2008; 97:1-20. [PubMed: 18479844]

Field M, Mogg K, Zetteler J, Bradley BP. Attentional biases for alcohol cues in heavy and light social drinkers: the roles of initial orienting and maintained attention. Psychopharmacology (Berl). 2004; 176:88-93. [PubMed: 15071718]

Fishbain DA, Cole B, Lewis J, Rosomoff HL, Rosomoff RS. What Percentage of Chronic Nonmalignant Pain Patients Exposed to Chronic Opioid Analgesic Therapy Develop Abuse/ 
Addiction and/or Aberrant Drug-Related Behaviors? A Structured Evidence-Based Review. Pain Medicine. 2007; 9(4):444-459. [PubMed: 18489635]

Garland EL, Carter K, Ropes K, Howard MO. Thought suppression, impaired regulation of urges, and Addiction-Stroop predict affect-modulated cue-reactivity among alcohol dependent adults. Biological Psychology. 2012; 89(1):87-93. [PubMed: 21967855]

Garland EL, Franken IH, Howard MO. Cue-elicited heart rate variability and attentional bias predict alcohol relapse following treatment. Psychopharmacology. 2012; 222(1):17-26. [PubMed: 22203318]

Garland EL, Franken IH, Sheetz JJ, Howard MO. Alcohol Attentional Bias Is Associated With Autonomic Indices of Stress-Primed Alcohol Cue-Reactivity in Alcohol-Dependent Patients. Experimental and Clinical Psychopharmacology. 201210.1037/a0027199

Garland EL, Froeliger BE, Passik SD, Howard MO. Attentional bias for prescription opioid cues among opioid dependent chronic pain patients. Journal of Behavioral Medicine. 2013b; 36(6):611620. [PubMed: 22968666]

Garland EL, Froeliger B, Howard MO. Effects of Mindfulness-Oriented Recovery Enhancement on reward responsiveness and opioid cue-reactivity. Psychopharmacology. 2014a:1-10.

Garland EL, Froeliger B, Howard MO. Neurophysiological evidence for remediation of reward processing deficits in chronic pain and opioid misuse following treatment with MindfulnessOriented Recovery Enhancement: Exploratory ERP findings from a pilot RCT. Journal of Behavioral Medicine. 2014a

Garland EL, Froeliger B, Zeidan F, Partin K, Howard MO. The downward spiral of chronic pain, prescription opioid misuse, and addiction: Cognitive, affective, and neuropsychopharmacologic pathways. Neuroscience \& Biobehavioral Reviews. 2013a; 37(10, Part 2):2597-2607. [PubMed: 23988582]

Garland EL, Hanley A, Thomas EA, Knoll P, Ferraro J. Mindlessness predicts self-medication of negative emotion with prescription opioids. Manuscript Submitted for Publication. in press.

Gipson CD, Kupchik YM, Shen H, Reissner KJ, Thomas CA, Kalivas PW. Relapse induced by cues predicting cocaine depends on rapid, transient synaptic potentiation. Neuron. 2013; 77(5):867872. [PubMed: 23473317]

Heinz A, Siessmeier T, Wrase J, Hermann D, Klein S, Grusser SM, Bartenstein P. Correlation between dopamine $\mathrm{D}(2)$ receptors in the ventral striatum and central processing of alcohol cues and craving. Am J Psychiatry. 2004; 161:1783-9. [PubMed: 15465974]

Inagaki H, Kuwahara M, Tsubone H. Changes in autonomic control of heart associated with classical appetitive conditioning in rats. Expm Anim. 2005; 54:61-9.

Ingjaldsson JT, Laberg JC, Thayer JF. Reduced heart rate variability in chronic alcohol abuse: relationship with negative mood, chronic thought suppression, and compulsive drinking. Biological Psychiatry. 2003a; 54:1427-36. [PubMed: 14675808]

Ingjaldsson JT, Laberg JC, Thayer JF. Reduced heart rate variability in chronic alcohol abuse: relationship with negative mood, chronic thought suppression, and compulsive drinking. Biological Psychiatry. 2003b; 54:1427-36. [PubMed: 14675808]

Kalivas PW, Volkow ND. The neural basis of addiction: a pathology of motivation and choice. Am J Psychiatry. 2005; 162:1403-13. [PubMed: 16055761]

Koob GF, Le Moal M. Drug addiction, dysregulation of reward, and allostasis. Neuropsychopharmacology. 2001; 24:97-129. [PubMed: 11120394]

Koob GF, Le Moal M. Addiction and the brain antireward system. Annu Rev Psychol. 2008; 59:29_ 53. [PubMed: 18154498]

Koob GF, Volkow ND. Neurocircuitry of addiction. Neuropsychopharmacology. 2009; 35(1):217238. [PubMed: 19710631]

Lang P, Bradley M, Cuthbert B. International affective picture system (IAPS): Technical manual and affective ratings. NIMH Center for the Study of Emotion and Attention. 1997

Lintas A, Chi N, Lauzon NM, Bishop SF, Sun N, Tan H, Laviolette SR. Inputs from the basolateral amygdala to the nucleus accumbens shell control opiate reward magnitude via differential dopamine D1 or D2 receptor transmission. European Journal of Neuroscience. 2012; 35(2):279_ 290. [PubMed: 22236063] 
Lubman DI, Allen NB, Peters LA, Deakin JF. Electrophysiological evidence of the motivational salience of drug cues in opiate addiction. Psychol Med. 2007; 37:1203-9. [PubMed: 17274853]

Lubman DI, Allen NB, Peters LA, Deakin JF. Electrophysiological evidence that drug cues have greater salience than other affective stimuli in opiate addiction. J Psychopharmacol. 2008; 22:83642. [PubMed: 18208907]

Lubman DI, Peters LA, Mogg K, Bradley BP, Deakin JF. Attentional bias for drug cues in opiate dependence. Psychol Med. 2000; 30:169-75. [PubMed: 10722187]

Lubman DI, Yucel M, Kettle JW, Scaffidi A, Mackenzie T, Simmons JG, Allen NB. Responsiveness to drug cues and natural rewards in opiate addiction: associations with later heroin use. Arch Gen Psychiatry. 2009; 66:205-12. [PubMed: 19188543]

Martel, Dolman, Edwards RR, Jamison RN, Wasan AD. The association between negative affect and prescription opioid misuse in patients with chronic pain: The mediating role of opioid craving. The Journal of Pain. 2014; 15(1):90-100. [PubMed: 24295876]

Malliani A, Lombardi F, Pagani M. Power spectrum analysis of heart rate variability: A tool to explore neural regulatory mechanisms. British Heart Journal. 1994; 71(1):1-2. [PubMed: 8297682]

Meltzer EC, Rybin D, Saitz R, Samet JH, Schwartz SL, Butler SF, Liebschutz GM. Identifying prescription opioid use disorder in primary care: diagnostic characteristics of the Current Opioid Misuse Measure (COMM). Pain. 2011; 152(2):397-402.10.1016/j.pain.2010.11.006 [PubMed: 21177035]

Nederkoorn C, Smulders FTY, Jansen A. Cephalic phase responses, craving and food intake in normal subjects. Appetite. 2000; 35(1):45-55.10.1006/appe.2000.0328 [PubMed: 10896760]

Nes LS, Roach AR, Segerstrom SC. Executive functions, self-regulation, and chronic pain: A review. Annals of Behavioral Medicine. 2009; 37:173-183. [PubMed: 19357933]

Rajan I, Murthy PJ, Ramakrishnan AG, Gangadhar BN, Janakiramaiah N. Heart rate variability as an index of cue reactivity in alcoholics. Biological Psychiatry. 1998; 43:544-6. [PubMed: 9547935]

Ratcliffe R. Methods of dealing with reaction-time outliers. Psychological Bulletin. 1993; 114:510532. [PubMed: 8272468]

Schoth DE, Nunes VD, Liossi C. Attentional bias towards pain-related information in chronic pain: A meta-analysis of visual probe investigations. Clinical Psychology Review. 2012; 32(1):13-25. [PubMed: 22100743]

Schwartz N, Temkin P, Jurado S, Lim BK, Heifets BD, Polepalli JS, Malenka RC. Decreased motivation during chronic pain requires long-term depression in the nucleus accumbens. Science. 2014; 345(6196):535-542. [PubMed: 25082697]

Segerstrom SC, Nes LS. Heart rate variability reflects self-regulatory strength, effort, and fatigue. Psychological Science. 2007; 18:275-281. [PubMed: 17444926]

Shurman J, Koob GF, Gutstein HB. Opioids, pain, the brain, and hyperkatifeia: a framework for the rational use of opioids for pain. Pain Medicine (Maiden, Mass). 2010; 11(7):1092-1098.10.1111/j. 1526-4637.2010.00881.x

Stockhorst U, Huenig A, Ziegler D, Scherbaum WA. Unconditioned and conditioned effects of intravenous insulin and glucose on heart rate variability in healthy men. Physiol Behav. 2011; 103:31-8. [PubMed: 21256144]

Thayer JF, Lane RD. A model of neurovisceral integration in emotion regulation and dysregulation. Journal of Affective Disorders. 2000; 61:201-16. [PubMed: 11163422]

Thayer JF, Lane RD. Claude Bernard and the heart-brain connection: further elaboration of a model of neurovisceral integration. Neuroscience and Biobehavioral Reviews. 2009; 33:81-8. [PubMed: 18771686]

Udo, T.; Weinberger, AH.; Grilo, CM.; Brownell, KD.; DiLeone, RJ.; Lampert, R.; McKee, SA. Heightened vagal activity during high-calorie food presentation in obese compared with non-obese individuals—Results of a pilot study. Obesity Research \& Clinical Practice. 2013. Retrieved from http://www.sciencedirect.com/science/article/pii/S1871403X13000458

Volkow ND, Wang GJ, Fowler JS, Tomasi D, Telang F. Addiction: Beyond dopamine reward circuitry. Proceedings of the National Academy of Sciences. 2011; 108(37):15037-15042.

Wadlinger HA, Isaacowitz DM. Fixing our focus: training attention to regulate emotion. Pers Soc Psychol Rev. 2010; 15:75-102. [PubMed: 20435804] 
- As addiction progresses, natural reward processing becomes dysregulated

- Prescription opioid misusers may exhibit deficits in natural reward processing

- Opioid misusers exhibited reduced HRV while attending to natural reward cues

- Unlike misusers, non-misusers have an attentional bias towards natural reward cues

- Reduced reward processing may indicate opioid misuse risk in chronic pain patients 


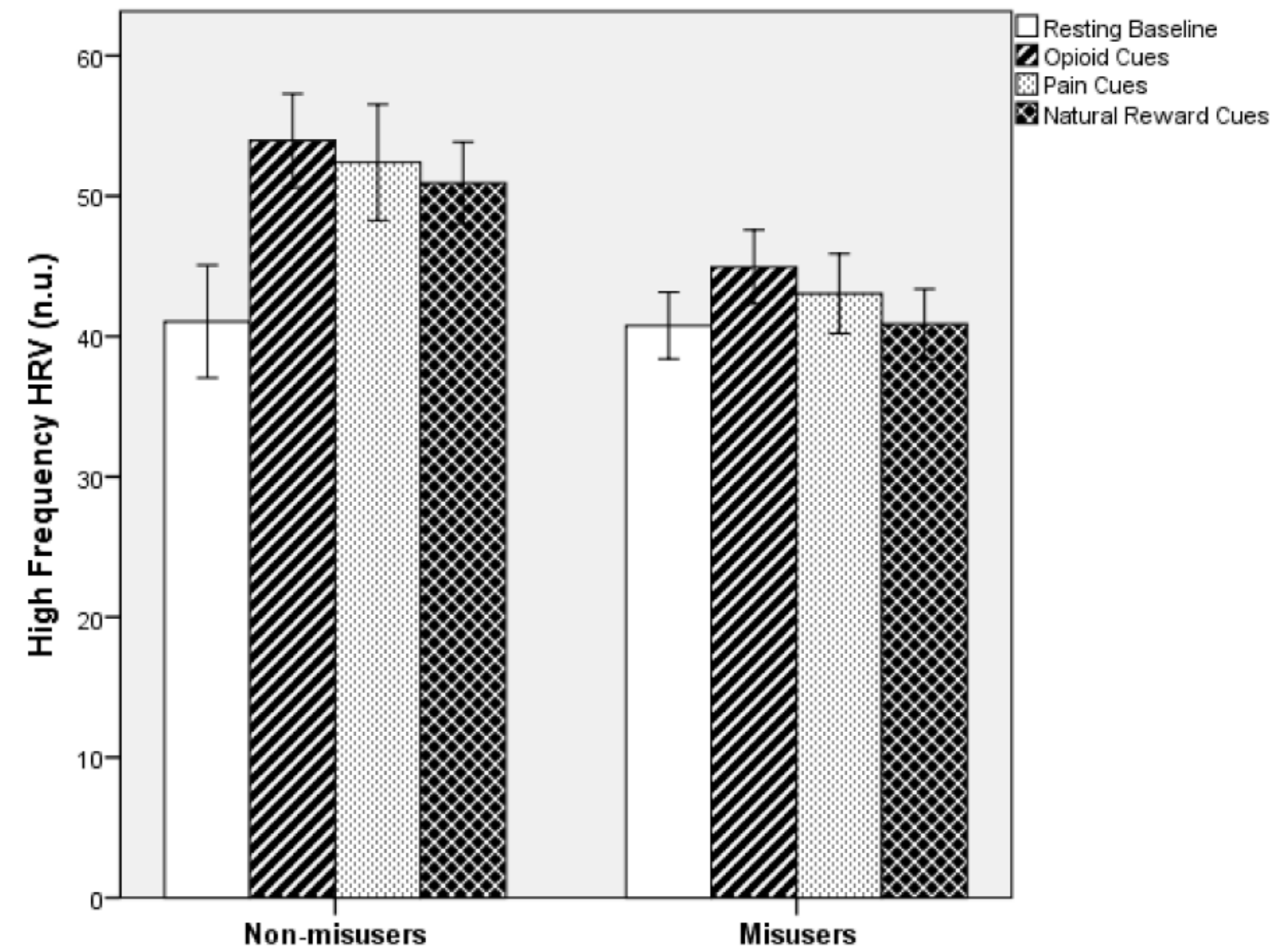

Figure 1.

High frequency heart rate variability (HRV) responses in normalized units (n.u.) at resting baseline and during each of three blocks of cues on a dot probe task among a sample of prescription opioid misusing chronic pain patients (misusers, $\mathrm{n}=72$ ) and those at low risk for prescription opioid misuse (non-misusers, $n=26$ ). Error bars indicate \pm 1 standard error. 


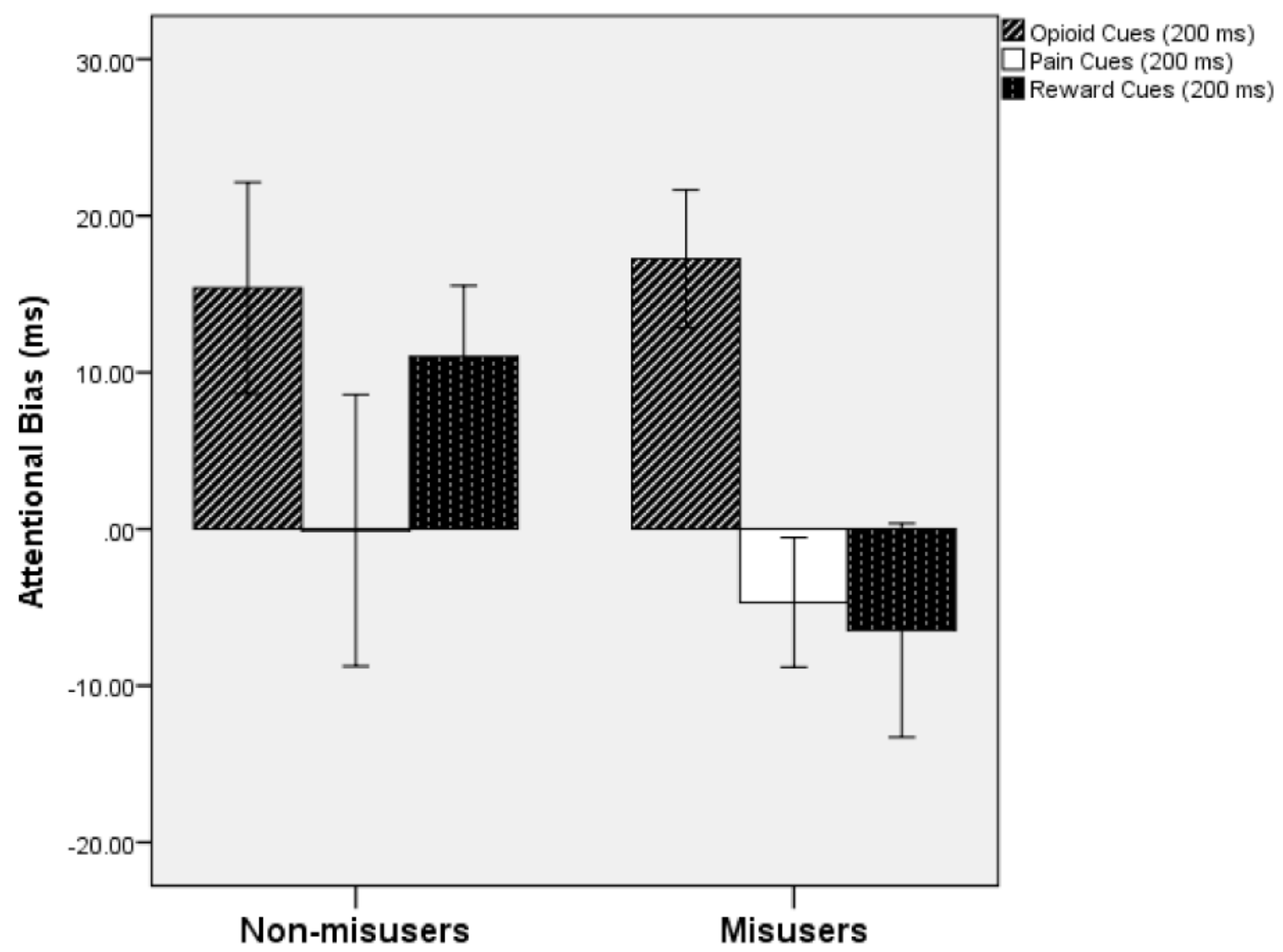

Figure 2.

Attentional bias (AB) scores for opioid, pain-related, and natural reward cues presented for 200 ms during a dot probe task among a sample of prescription opioid misusing chronic pain patients (misusers, $\mathrm{n}=72$ ) and those at low risk for prescription opioid misuse (nonmisusers, $n=26$ ). Positive scores indicate a bias towards the emotionally-salient cue; negative scores indicate a bias away from the emotional cue. Error bars indicate \pm 1 standard error. 


\section{Table 1}

Sample characteristics of opioid misusers $(n=72)$ and non-misusers $(n=26)$ in the study.

\begin{tabular}{|c|c|c|}
\hline & $\begin{array}{l}\text { Opioid Misusers } \\
(n=72)\end{array}$ & $\begin{array}{l}\text { Non-Misusers } \\
(n=26)\end{array}$ \\
\hline Age (years) & $46.6(\mathrm{SD}=13.4)$ & $47.3(\mathrm{SD}=11.6)$ \\
\hline Gender (women, \%) & $49(69.2 \%)$ & $18(68.1 \%)$ \\
\hline \multicolumn{3}{|l|}{ Chronic pain conditions ${ }^{a}$ : } \\
\hline Lumbago & $43(59.7 \%)$ & $13(50.0 \%)$ \\
\hline Fibromyalgia & $13(18.0 \%)$ & $7(26.9 \%)$ \\
\hline Arthritis & $5(6.9 \%)$ & $2(7.7 \%)$ \\
\hline Cervicalgia & $5(6.9 \%)$ & $0(0.0 \%)$ \\
\hline Other & $6(8.3 \%)$ & $4(15.3 \%)$ \\
\hline Duration of chronic pain (years) & $10.7(11.0)$ & $12.7(9.5)$ \\
\hline Opioid misuse score (COMM) & $21.9(9.2)$ & $8.5(3.1)$ \\
\hline
\end{tabular}

${ }^{a}$ Note: Participants could report more than one chronic pain condition. 